\title{
Oleuropein induces apoptosis via activation of caspases and suppression of phosphatidylinositol 3-kinase/protein kinase $B$ pathway in HepG2 human hepatoma cell line
}

\author{
CHUN-MEI YAN $^{1 *}$, ER-QING CHAI ${ }^{2 *}$, HONG-YI CAI $^{1}$, GUO-YING MIAO $^{1}$ and WEN MA ${ }^{1}$ \\ ${ }^{1}$ Department of Radiotherapy Oncology; ${ }^{2}$ Cerebrovascular Diseases Center, \\ Gansu Provincial Hospital, Lanzhou, Gansu 730000, P.R. China
}

Received March 26, 2014; Accepted December 12, 2014

DOI: $10.3892 / \mathrm{mmr} .2015 .3266$

\begin{abstract}
Oleuropein is a polyphenol, that is found in extra-virgin olive oil. Previous studies have shown that oleuropein inhibits cell proliferation and induces apoptosis in breast cancer, colorectal cancer and thyroid cancer. The aim of the present study was to investigate the effects of oleuropein in hepatocellular carcinoma (HCC) cells. The results of Cell Counting Kit 8 and flow cytometric analysis indicated that oleuropein effectively inhibited cell viability and induced apoptosis in HepG2 human hepatoma cells in a dose-dependent manner, through activation of the caspase pathway. Proapoptotic Bcl-2 family members, BAX and Bcl-2, were involved in oleuropein-induced apoptosis. The phosphatidylinositol 3-kinase/protein kinase B (PI3K/AKT) signaling pathway was also shown to be involved in this process. Oleuropein was demonstrated to suppress the expression of activated AKT. In addition, AKT overexpression promoted cell survival following treatment with oleuropein, while inhibition of AKT promoted cell death. Furthermore, the data demonstrated that oleuropein induces the production of reactive oxygen species (ROS) and that the function of oleuropein is, at least partially, ROS-dependent. These results suggest that
\end{abstract}

Correspondence to: Ms. Chun-Mei Yan, Department of Radiotherapy Oncology, Gansu Provincal Hospital, 204 Donggangxi Road, Lanzhou, Gansu 730000, P.R. China

E-mail: yanchm123@126.com

*Contributed equally

Abbreviations: HCC, hepatocellular carcinoma; ROS, reactive oxygen species; BAX, B cell lymphoma 2-associated $\mathrm{X}$ protein; Bcl-2, B cell lymphoma 2; mRNA, messenger RNA; NF- $\kappa$ B, Nuclear Factor- $\kappa \mathrm{B}$; PI3K, phosphatidylinositol-4,5-bisphosphate 3-kinase; AKT, serine/threonine protein kinase $\mathrm{B}$; mTOR, mechanistic target of rapamycin; iNOS, inducible nitric oxide synthases

Key words: oleuropein, hepatocellular carcinoma, proliferation, apoptosis, protein kinase B oleuropein may be a promising novel chemotherapeutic agent in hepatocellular carcinoma.

\section{Introduction}

Hepatocellular carcinoma (HCC) is the third most common cause of cancer-related mortality (1). The predominant risk factors for $\mathrm{HCC}$ development are infection with hepatitis $\mathrm{B}$ or $\mathrm{C}$ virus, obesity and excess alcohol intake. The incidence and mortality of HCC is increasing as a result of the current obesity epidemic and rise in alcohol consumption (2). However, only $10-20 \%$ of patients with HCC are eligible for surgical resection, due to poorly preserved liver function, portal vein invasion or extrahepatic spread. Furthermore, the risk of recurrence following HCC resection is high $(3,4)$. The available chemotherapeutic and radiotherapeutic treatment options for patients with advanced HCC are also extremely limited. Therefore, it is necessary to develop effective and practical chemotherapeutic agents with minimal cytotoxicity for use in this disease.

A number of previous studies have shown that regular consumption of coffee, vitamin E and fish oil may be associated with a reduced risk of developing HCC (5-7). Ecological studies have investigated the association between dietary fat and certain types of cancer $(8,9)$. Hursting et al (8) demonstrated that the intake of saturated or polyunsaturated fats was associated with incidence of breast and prostate cancer. A causal relationship was identified between cholesterol intake and colon cancer (9). Olive oil is the oil obtained from the fruit of the olive tree (Olea europaea Sativa) and its consumption is associated with lower overall mortality patterns, which are observed in Mediterranean populations (10). The primary component of olive leaf extract is oleuropein. Andreadou et al reported that oleuropein is involved in cardiomyocyte metabolism through the activation of AMP-activated protein kinase $\alpha$ (AMPK) and suppression of inducible nitric oxide synthases (iNOS) (11). It has recently been reported that oleuropein may have therapeutic potential in a number of types of cancer, including breast cancer (12), thyroid cancer (13) and colorectal cancer (14), via inhibition of cell growth induction of apoptosis. However, to date the effects of oleuropein on liver cancer remain largely unknown. 
In multicellular organisms, apoptosis is a tightly-regulated multi-step pathway that is responsible for the process of cell death (15). The majority of chemotherapies function as tumor suppressors by targeting this pathway (16). Serine/threonine protein kinase B (AKT), is important in cell proliferation, apoptosis and cell survival.

Aberrant and excessive activation of phosphatidylinositol 3-kinase (PI3K)/AKT signaling is a hallmark of a number of types of human cancer $(17,18)$. AKT mediates PI3K-dependent cell survival by phosphorylating a variety of substrates, such as glycogen synthase kinase- $3 \beta, 6$-phosphofructo- 2 kinase and apoptosis signal-regulating kinase 1 (19). Changes in the biological activity of specific downstream substrates of AKT affect whole signaling cascades, including the activity of transcription factors involved in cell proliferation, apoptosis, inflammation and metastasis (20-22).

To understand the possible role of oleuropein in hepatocellular carcinoma, CCK8 and flow cytometry assays were used to evaluate HepG2 cell viability and apoptosis following oleuropein treatment. A luciferase assay was subsequently used to effectively identify signaling pathways modulated by oleuropein in liver cancer cells. The results of the present study may identify the therapeutic potential of oleuropein for the treatment of liver cancer.

\section{Materials and methods}

Cell culture and treatment. The HepG2 and Huh7 human hepatocellular carcinoma cell lines and the RKO human colorectal cancer cell line were obtained from the American Type Culture Collection (ATCC, Manassas, VA, USA), and maintained in McCoy's 5A medium (Applichem, Darmstadt, Germany) supplemented with $10 \%$ fetal bovine serum (Gibco Life Technologies, Carlsbad, CA, USA) and $1 \%$ penicillin-streptomycin (Invitrogen Life Technologies, Carlsbad, CA, USA). Cells were incubated in an atmosphere of $5 \% \mathrm{CO}_{2}$ at $37^{\circ} \mathrm{C}$. Cells were treated in serum-free medium with the indicated concentrations of oleuropein (Sigma-Aldrich, St. Louis, MO, USA; 12247), dimethyl sulfoxide (Sigma-Aldrich; D2650) and glutathione (GSH; Sigma-Aldrich; V900456).

Cell viability assay. The effects of oleuropein on cell viability were determined using a Dojindo Cell Counting kit-8 (CCK-8; Dojindo Molecular Technologies, Kumamoto, Japan). HepG2, Huh7 and RKO cells were cultured in 96-well plates at approximately $10^{4}$ cells per well for $24 \mathrm{~h}$ at $37^{\circ} \mathrm{C}$. Cells were treated with $(0,20,40,60,80$ or $100 \mu \mathrm{M})$ oleuropein for $24 \mathrm{~h}$. Subsequently, $10 \mu \mathrm{l}$ of CCK-8 solution was added to each well and incubated for $1 \mathrm{~h}$ at $37^{\circ} \mathrm{C}$. The absorbance at $450 \mathrm{~nm}$ was measured using a spectrophotometer (Shimadzu UV-1800; Shimadzu Scientific Instruments, Columbia, MD, USA). Independent experiments were performed three times in triplicate.

Colony formation assay. HepG2 cells (2000 cells/well) were seeded into six-well plates (in triplicate). Cells were permitted to adhere for $24 \mathrm{~h}$ at $37^{\circ} \mathrm{C}$, following which the media was changed for media mixed with $(0,20,40,60,80$ or $100 \mu \mathrm{M})$ oleuropein. After $48 \mathrm{~h}$, cells were cultured in normal media for $10 \mathrm{~d}$. In order to visualize colonies, cells were fixed with
4\% paraformaldehyde (Sigma-Aldrich) for $15 \mathrm{~min}$ and stained with crystal violet staining solution (BD Biosciences, San Jose, CA, USA) for $30 \mathrm{~min}$. Colonies were counted and presented as the mean number of colonies formed from three independent experiments.

DNA fragmentation. HepG2 cells were harvested, washed with phosphate-buffered saline (PBS) and resuspended in $40 \mu \mathrm{l}$ lysis buffer, which contained $100 \mathrm{mM}$ Tris ( $\mathrm{pH}$ 8.0; Amresco LLC, Solon, OH, USA), 20 mM EDTA (Sinopharm, Shanghai, China), $0.8 \%$ sodium dodecyl sulfate (SDS; W/V; Sinopharm) and $0.5 \mathrm{mg} / \mathrm{ml}$ proteinase K (Merck Millipore, Darmstadt, Germany), at $65^{\circ} \mathrm{C}$ for $3 \mathrm{~h}$. RNase A $(0.5 \mathrm{mg} / \mathrm{ml}$; Sigma-Aldrich) was added to the cell lysates and incubated for a further $3 \mathrm{~h}$ at $55^{\circ} \mathrm{C}$. Genomic DNA was extracted using phenol-chloroformisoamyl alcohol (25:24:1), separated by $1 \%$ agarose gel electrophoresis and scanned with an Image Analyzer (Tanon-2500; Tanon Science and Technology Co., Ltd., Shanghai, China).

Detection of cell apoptosis by flow cytometry. Detection of apoptosis induced by oleuropein was performed using Annexin V and Propidium Iodide (PI) staining, according to the manufacturer's instructions (BD, Pharmingen San Diego, CA, USA). Cells $\left(1 \times 10^{5}\right)$ were suspended in $100 \mu$ l binding buffer and stained with Annexin V-PE and PI $(1 \mu \mathrm{g} / \mathrm{ml})$. Following incubation at room temperature, a further $400 \mu \mathrm{l}$ binding buffer was added and the apoptotic cells were quantification using flow cytometry (Becton Dickinson FACsCalibur ${ }^{\mathrm{TM}}$, BD Biosciences, Franklin Lakes, NJ, USA).

ROS measurement. HepG2 cells in a 24 -well plate were harvested following treatment for $24 \mathrm{~h}$. Cells were washed twice with PBS, labeled with $10 \mu \mathrm{M}$ 2,7-dichlorodihydrofluorescein diacetate (Sigma-Aldrich) at $37^{\circ} \mathrm{C}$ for $30 \mathrm{~min}$ in darkness, and then subjected to flow cytometry for the measurement of reactive oxygen species (ROS).

Luciferase assay. Signaling pathway arrays were conducted using a luciferase assay. Reporters, NF- $\kappa B-l u c$, PI3K/AKT-luc and Notch-luc, were purchased from Qiagen (Qiagen-SABiosciences, Valencia, CA, USA). HepG2 cells were cotransfected with reporter plasmids and a control reporter in 24 -well plates. After $48 \mathrm{~h}$, the luciferase activity of firefly and Renilla cells was measured using a dual Luciferase Reporter Assay system (Promega Corporation, Madison, WI, USA).

Quantitative polymerase chain reaction $(q P C R)$. Total RNA from HepG2 cells, with or without oleuropein treatment, was isolated using RNAiso Plus (Takara Bio, Inc., Otsu, Japan) according to the manufacturer's instructions. cDNA was synthesized using a PrimeScript ${ }^{\mathrm{TM}}$ RT-PCR kit (Takara Bio, Inc.). qPCR was performed using LightCycler (Roche, Basel, Switzerland) according to the manufacturer's recommendations. The following primer sequences (Sagon Biotech, Shanghai, China) were used: Forward: 5'-GAGGATGATTGCCGCCGTGGACA-3' and reverse: 5'-GGTGGGGGAGGAGGCTTGAGG-3' for BAX, forward: 5'-ATGTGTGTGGAGAGCGTCAACC-3' and reverse: 
A

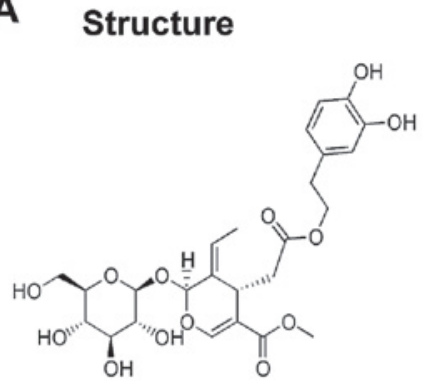

C

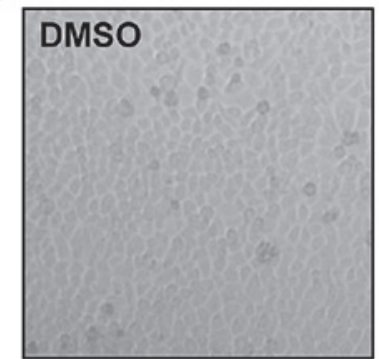

D Oleuropein $(\mu \mathrm{M})$

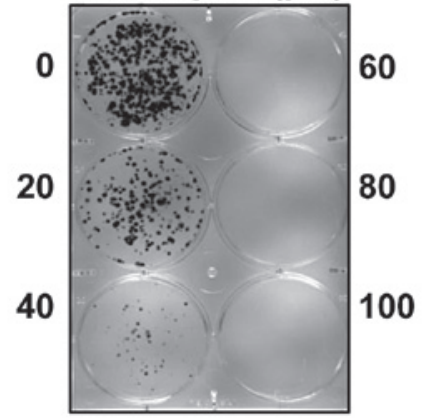

B

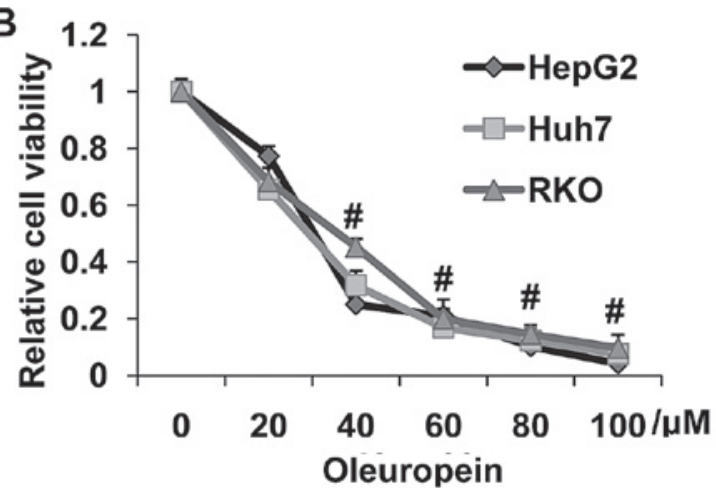

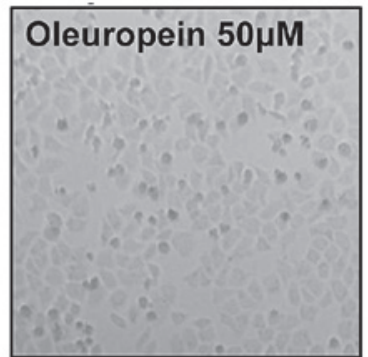

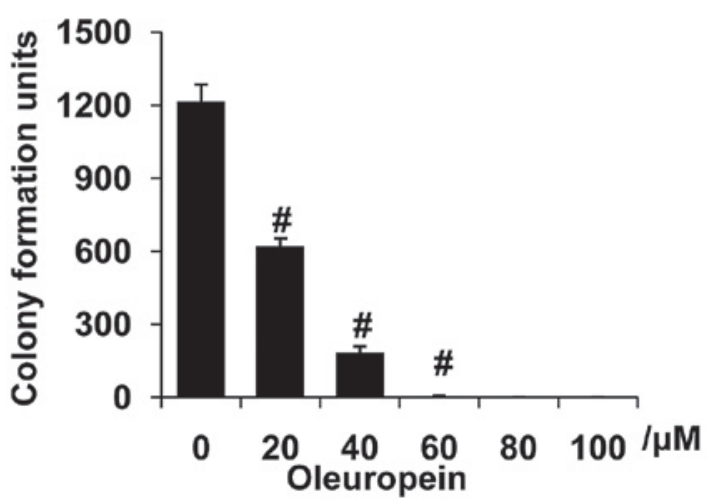

Figure 1. Effects of oleuropein on cell viability and proliferation of HCC cells. (A) Structure of Oleuropein. (B) HepG2, Huh7 and RKO cells were treated with oleuropein at different concentrations $(0,10,20,40,60$ and $80 \mu \mathrm{M})$ for $24 \mathrm{~h}$ and then supplemented with CCK- 8 to assess cell viability. ${ }^{\sharp} \mathrm{P}<0.05$ compared with the control group (DMSO treatment). (C) Representative light microscopy images showing morphology of HepG2 cells treated with oleuropein (0, 25 and $50 \mu \mathrm{M}$ ). (D) Oleuropein inhibited the proliferation of HepG2 cells in a colony formation assay. ${ }^{\text {P }}<0.05$ compared with the control group (DMSO treatment). Data are presented as the mean \pm standard deviation. HCC, hepatocellular carcinoma; CCK-8, cell counting kit-8; DMSO, dimethyl sulfoxide.

5'-TGAGCAGAGTCTTCAGAGACAGCC-3' for Bcl-2 and forward: 5'-TGCACCACCAACTGCTTAGC-3' and reverse: 5'-GCATGGACTGTGGTCATGAG-3' for GAPDH. All samples were read in triplicate, and values were normalized to the expression of GAPDH.

Western blot analysis. HepG2 cells, with or without oleuropein treatment, were lysed in ice-cold radioimmunoprecipitation assay buffer containing protease inhibitors $[20 \mathrm{mmol} / 1$ Tris, $150 \mathrm{mmol} / 1 \mathrm{NaCl}$ (Sinopharm), 1\% NP40 (Sigma-Aldrich), $0.5 \%$ sodium deoxycholate (Sigma-Aldrich), 1 mmol/1 EDTA, $1 \mathrm{mmol} / \mathrm{l}$ phenylmethylsulfonyl fluoride (Sigma-Aldrich), 0.15 units/ml aprotinin and $10 \mathrm{mmol} / \mathrm{l}$ leupeptin (Roche Diagnostics GmBH, Mannheim, Germany)]. Cells were lysed for $30 \mathrm{~min}$ on ice and lysates were then centrifuged at $11,340 \mathrm{x} \mathrm{g}$ for $10 \mathrm{~min}$ at $4^{\circ} \mathrm{C}$. The protein concentration was determined by a bicinchoninic acid protein assay kit (Pierce, 23227; Pierce Biotechnology, Inc., Rockford, IL, USA) according to the manufacturer's instructions. Lysates were dissolved in 4X loading buffer and boiled for $8 \mathrm{~min}$. Samples were separated by $12 \%$ SDS-PAGE and transferred onto a polyvinylidene fluoride membrane (EMD Millipore, Bedford, MA, USA). Samples were blocked with $5 \%$ non-fat milk in $50 \mathrm{mM}$ Tris-buffered saline ( $\mathrm{pH} 7.4$ ) with $0.1 \%$ Tween-20). Membranes were probed with primary antibodies for $3 \mathrm{~h}$, followed by a horseradish peroxidase-conjugated IgG (Thermo Fisher Scientific, Rockford, IL, USA). Bands were revealed using an enhanced chemiluminescence detection system (Immobilon Western Chemiluminescent HRP Substrate; EMD Millipore). The following primary antibodies were used: Rabbit monoclonal anti-poly ADP ribose polymerase (PARP) antibody (1:1,000; \#9532; Cell Signaling Technology, Inc., Danvers, MA, USA); rabbit polyclonal anti-caspase-9 antibody (1:1,000; \#9504; Cell Signaling Technology, Inc.); rabbit polyclonal anti-caspase-8 antibody (1:1,000; \#4927; Cell Signaling Technology, Inc.); mouse polyclonal anti-caspase-3 antibody $(1: 1,000$; \#9665; Cell Signaling Technology, Inc.); rabbit polyclonal anti-BAX antibody (1:1,000; \#2772; Cell Signaling Technology, Inc.); 

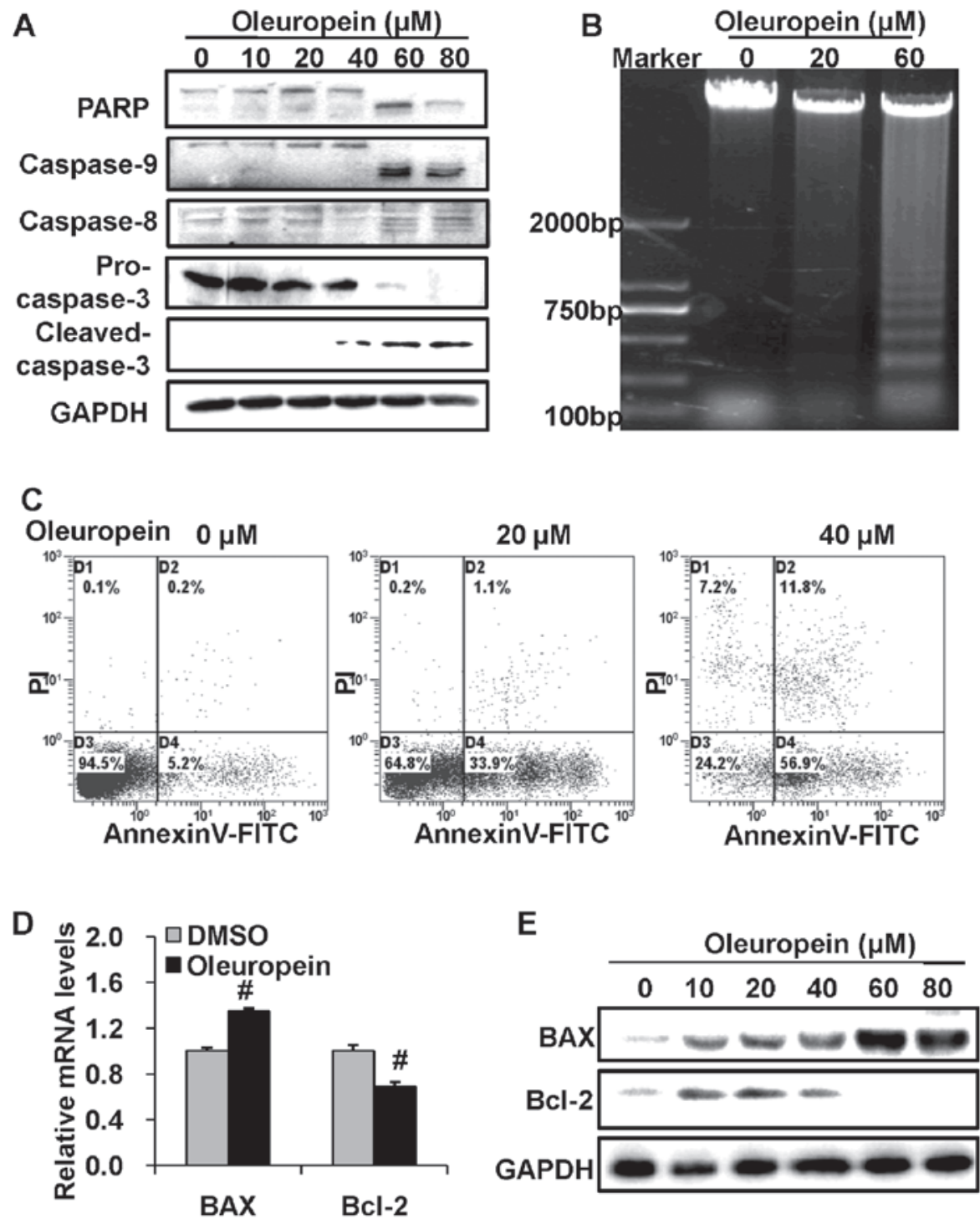

Figure 2. Cell apoptosis was induced by oleuropein. (A) Equal amounts of cell lysates was subjected to western blot analysis in order to detect PARP, Caspase-9, Caspase-8 and Caspase-3. (B) DNA fragmentation assay and (C) flow cytometry analysis confirmed that apoptosis was induced by oleuropein. (D) Oleuropein promoted the expression of BAX and reduced the expression of $\mathrm{Bcl}-2$, as shown by quantitative polymerase chain reaction. "P<0.05 compared with the control group (DMSO treatment). (E) Western blot analysis of the expression of BAX and Bcl-2 proteins in HepG2 cells at 0, 10, 20, 40,60 and 80 $\mu \mathrm{M}$ oleuropein. GAPDH served as an internal control. Data are presented as the mean \pm standard deviation. PARP, poly ADP ribose polymerase; DMSO, dimethyl sulfoxide; FITC, fluorescein isothiocyanate.

rabbit polyclonal anti-Bcl-2 antibody $(1: 1,000 ;$ \#2872; Cell Signaling Technology, Inc.); mouse monoclonal anti-flag antibody (1:2,000; \#3165; Sigma-Aldrich) and rabbit monoclonal anti-GAPDH antibody (1:5,000; \#3683; Cell Signaling Technology, Inc.).

Statistical analysis. Analysis of variance using SPSS 19.0 (IBM SPSS, Armonk, NY, USA) or Student's $t$-test were employed to analyze the data, $\mathrm{P}<0.05$ were considered to indicate a statistically significant difference.

\section{Results}

Oleuropein inhibits HepG2 cell growth in a dose-dependent manner. Oleuropein, whose structure is exhibited in Fig. 1A, was the bioactive polyphenol in olive oil. In order to determine whether oleuropein affects the tumorigenic properties of human HCC cells, HepG2 and Huh7 human hepatoma cells were treated with various concentrations of oleuropein. The human RKO colorectal cancer cell line was also treated with oleuropein under the same conditions. Using a cell viability assay, a significant dose-dependent inhibition of cell growth was observed when the concentration of oleuropein was in the 20-80 $\mu \mathrm{M}$ range (Fig. 1B). In addition, alterations in the morphology of HepG2 tumor cells, including cell shrinkage and increased cell death, were observed following treatment with oleuropein (Fig. 1C). Furthermore, the colony formation assay demonstrated a marked reduction in the number of colonies formed in the oleuropein-treated cells, when compared with the control cells (Fig. 1D). These findings suggest that oleuropein is involved in the growth of liver cancer cells.

Oleuropein induces cell apoptosis through activation of caspases. In order to examine the mechanisms involved 

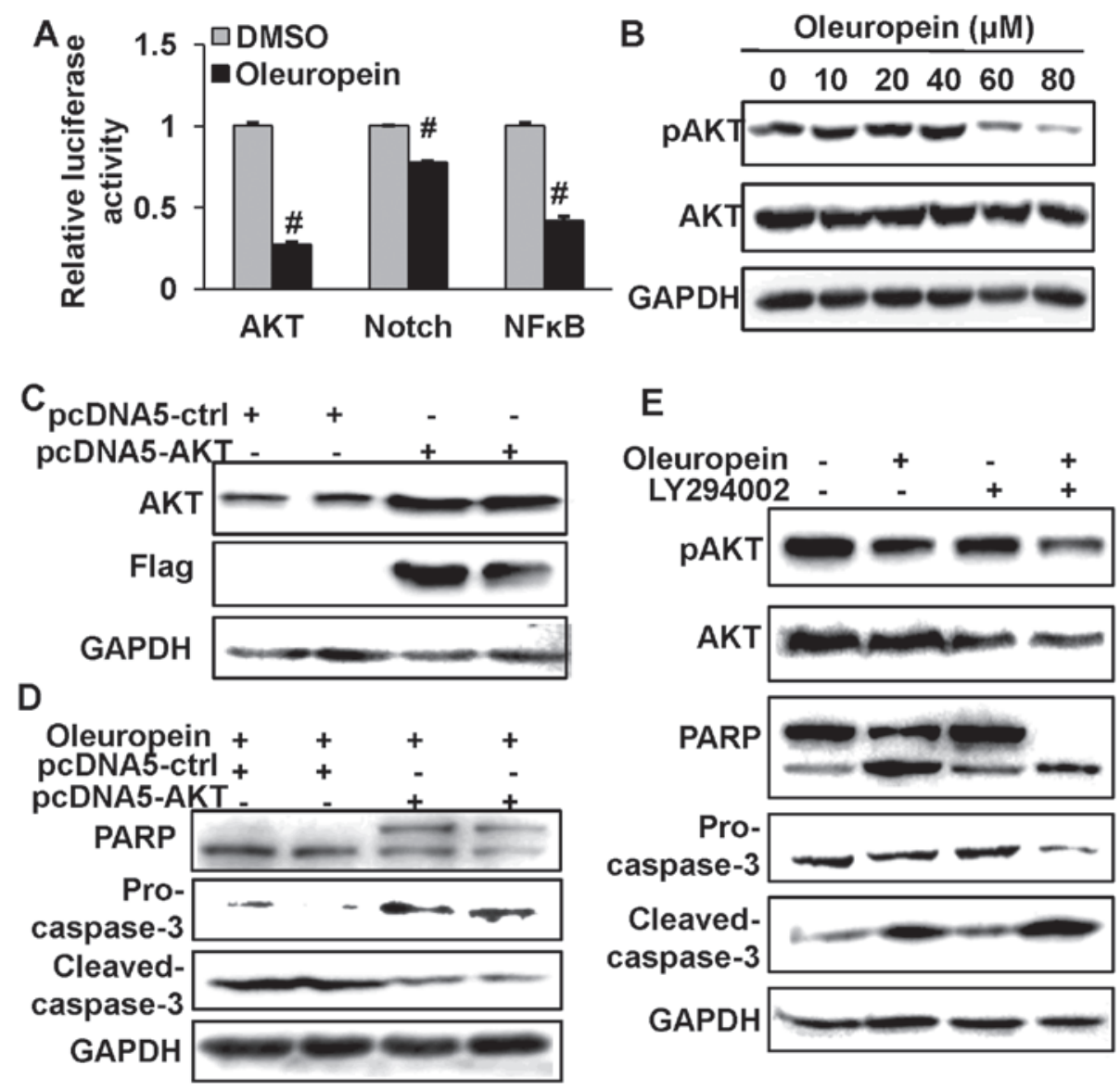

Figure 3. PI3K/AKT signaling pathway was involved in oleuropein-induced apoptosis. (A) Luciferase assay of the activity of AKT, Notch and NFkB, with or without $50 \mu \mathrm{M}$ oleuropein treatment. ${ }^{*} \mathrm{P}<0.05$ compared with the control group (DMSO treatment) (B) Western blot analysis of the levels of pAKT and AKT at $0,10,20,40,60$ and $80 \mu \mathrm{M}$ oleuropein. (C) Western blot analysis confirmed overexpression of flag-AKT in HepG2 cells. HepG2 cells were transfected with $2 \mu \mathrm{g}$ pcDNA5-flag-AKT or pcDNA5 empty vector. (D) AKT overexpression decreased the cleavage of PARP and caspase-3, as shown by western blot analysis. Cells were transfected with $2 \mu \mathrm{g}$ pcDNA5-flag-AKT or pcDNA5 empty vector for $24 \mathrm{~h}$ and treated with $50 \mu \mathrm{M}$ oleuropein. (E) Western blot analysis of the levels of the pAKT, AKT, PARP and caspase-3 proteins in HepG2 cells pretreated with $50 \mu \mathrm{M}$ LY294002 for $2 \mathrm{~h}$ and followed by $50 \mu \mathrm{M}$ oleuropein. GAPDH served as an internal control. Data are presented as the mean \pm standard deviation. PI3K, phosphatidylinositol 3-kinase; AKT, protein kinase B; NFkB, nuclear factor-кB; pAKT, phosphorylated AKT; PARP, poly ADP ribose polymerase.

in growth inhibition following treatment with oleuropein, the effect of oleuropein on the cascade of caspases was investigated. Caspases are essential initiators or effectors in cell apoptosis. The results of the present study showed that oleuropein induces cleavage of caspase-8, caspase-9, caspase-3 and PARP, which is a nuclear enzyme with a wide range of functions, including the regulation of DNA repair, cell differentiation and gene expression (Fig. 2A). To further confirm the involvement of apoptotic pathways in cell death due to oleuropein, a DNA fragmentation assay was conducted. A higher level of DNA fragmentation was observed in oleuropein-treated cells compared with control cells (Fig. 2B). Furthermore, cell apoptosis was assessed by Annexin V-FITC/PI staining in HepG2 cells treated with $30 \mu \mathrm{M}$ oleuropein. Following $48 \mathrm{~h}$ of treatment, a significant percentage of HepG2 cells were observed to be undergoing apoptosis (Fig. 2C). The Bcl-2 family of mitochondria proteins are involved in the regulation of apoptosis (23). In order to determine whether Bcl-2 family members are involved in oleuropein-induced apoptosis, the expression of BAX and Bcl-2 was measured. The results indicated that oleuropein treatment leads to the upregulation of the expression of
BAX mRNA and protein and the downregulation of that of Bcl-2 (Fig. 2D and E). This suggests that oleuropein induces HCC cell apoptosis and that this progress is mediated via the activation of caspases, in addition to the regulation of the expression of mitochondrial proteins.

Oleuropein induces apoptosis via suppression of the PI3K/AKT signaling pathway. In order to explore the primary regulators involved in oleuropein-induced apoptosis, a pathway array was performed using a luciferase assay. A significant reduction in the activity of (PI3K/AKT; Fig. 3A) was observed. AKT is involved in cell proliferation, apoptosis and cell survival. The activation of AKT by phosphorylation is associated with the protection of cells from apoptosis (24). The level of the phosphorylated AKT (S473) and total AKT proteins were analyzed. In accordance with the results from the luciferase assay, a high concentration of oleuropein $(60 \mu \mathrm{M})$ reduced the level of phosphorylated active AKT, while the level of total AKT remained unchanged with the various concentrations used (Fig. 3B).

To examine the role of AKT in oleuropein-induced apoptosis, a rescue experiment was conducted, in which HepG2 cells 
A DMSO

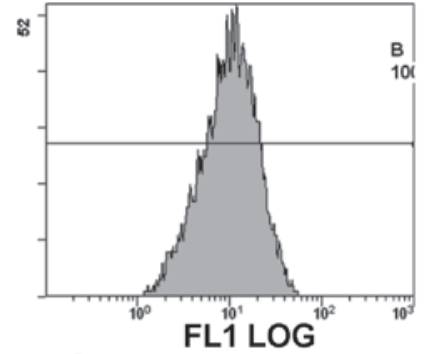

Oleuropein

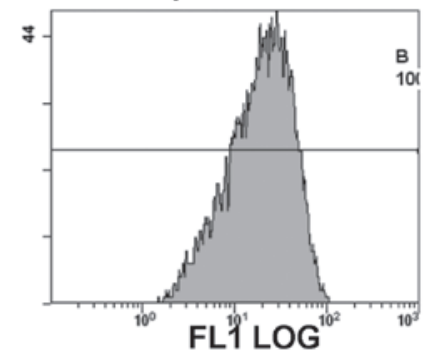

C

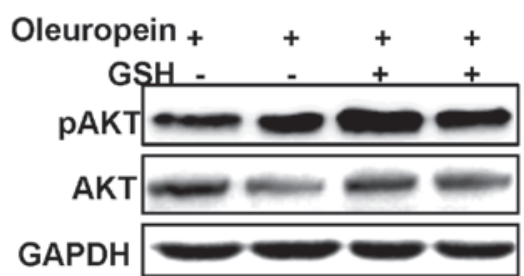

B
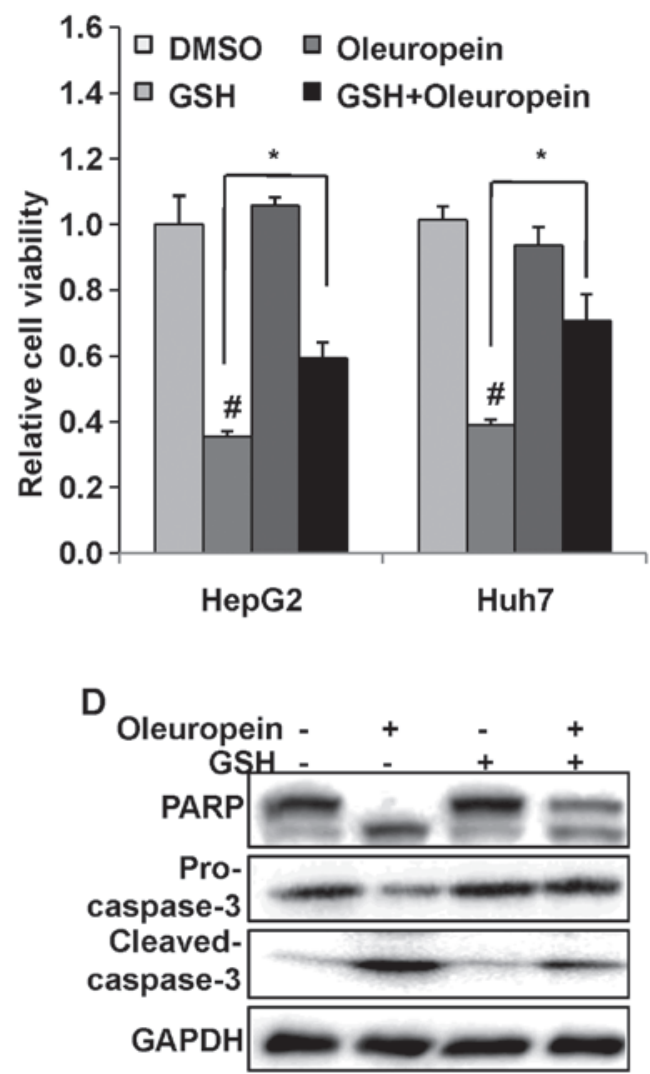

Figure 4. ROS generation was induced by oleuropein in HepG2 cells. (A) HepG2 cells treated with $50 \mu \mathrm{M}$ oleuropein or DMSO were subjected to flow cytometry in order to measure ROS production. (B) Cell viability assay of HepG2 cell treated with $50 \mu \mathrm{M}$ oleuropein or pretreated with $20 \mathrm{mM}$ GSH for $2 \mathrm{~h}$, and followed by $50 \mu \mathrm{M}$ oleuropein. ${ }^{~} \mathrm{P}<0.05$ compared with the control group (DMSO treatment). "P $<0.05$, GST pretreatment followed by oleuropein treatment compared with oleuropein treatment alone. (C) Western blot analysis of the expression of pAKT and AKT, and (D) apoptosis-related proteins, PARP and caspase-3, in HepG2 cells pretreated with $20 \mathrm{mM} \mathrm{GSH}$ for $2 \mathrm{~h}$ followed by $50 \mu \mathrm{M}$ oleuropein. GAPDH served as an internal control. Data are presented as the mean \pm standard deviation. ROS, reactive oxygen species; DMSO, dimethyl sulfoxide; GSH, glutathione; AKT, protein kinase B; pAKT, phosphorylated AKT; PARP, poly ADP ribose polymerase.

were transfected with pcDNA5-flag-AKT or pcDNA5-control constructs prior to treatment with oleuropein. The results indicated that AKT overexpression (Fig. 3C) causes less cleavage of PARP and caspase-3 compared with intrinsic AKT (Fig. 3D). LY294002, a specific PI3K inhibitor (25), led to increased cleavage of PARP and caspase-3 when used together with oleuropein (Fig. 3E). These results suggest that the PI3K/AKT signaling pathway contributes to oleuropein-induced apoptosis in HCC cells.

Oleuropein promotes the generation of ROS. It has been reported that AKT activation induces the production of ROS. Elevated levels of intracellular ROS contribute to tumorigenesis by activation of signaling pathways and by increasing mutation rates $(26,27)$. In order to determine whether the dose-dependent inhibition of AKT activation decreases the accumulation of intracellular ROS, HepG2 cells were treated with $50 \mu \mathrm{M}$ oleuropein for $48 \mathrm{~h}$ and the level of ROS was measured by FACS analysis. ROS accumulation was observed following $24 \mathrm{~h}$ of oleuropein treatment in HepG2 cells, as shown in Fig. 4A. We hypothesized that ROS generation resulted in PI3K/AKT inactivation. ROS blockers were then used to investigate this hypothesis. HepG2 cells were pretreated with $20 \mathrm{mM}$ GSH for $2 \mathrm{~h}$, followed by $50 \mu \mathrm{M}$ oleuropein treatment for an additional
$24 \mathrm{~h}$. A cell viability assay demonstrated that GSH pretreatment promoted cell survival (Fig. 4B). The results of the western blot analysis showed that the expression of phosphorylated AKT was increased following the removal of ROS by pretreatment with GSH (Fig. 4C). Pretreatment with GSH consistently inhibited the oleuropein-induced cleavage of PARP and caspase-3 (Fig. 4D). These results indicate that oleuropein-induced ROS generation leads to PI3K/AKT inactivation and is thus has an important function in the apoptotic pathway.

\section{Discussion}

A number of previous epidemiological studies have indicated that there is an association between the consumption of olive oil, and lower overall mortality patterns and risk of developing cancer (28-30). Oleuropein is one of the primary components of olive oil. The present study reports that oleuropein inhibited proliferation and induced apoptosis in HCC. Mechanistically, oleuropein-induced apoptosis appears to be mediated by suppression of the PI3K/AKT pathway and the production of ROS. It is suggested that oleuropein may be a promising candidate for HCC treatment.

Recent studies have reported that oleuropein inhibits cell proliferation and induces the apoptosis of cancer cell lines 
through diverse mechanisms. Cardeno et al (14) reported that oleuropein limited cell growth and induced apoptosis in HT29 colorectal cancer cells through a p53-dependent pathway (14). A similar mechanism was also observed in breast cancer cells (12). Additionally, a recent study on cardiomyopathy demonstrated that oleuropein has a protective effect in doxorubicin-induced cardiomyopathy through the activation of AMPK and suppression of iNOS (11). In the current study, evidence is presented for a role of oleuropein as an inhibitor of cell growth and an inducer of apoptosis, which suggests that oleuropein possesses antitumor activity in HCC.

Apoptosis is a common mechanism by which chemotherapies induces tumor cell death $(31,32)$. In mammals, two mechanisms are known to directly initiate apoptosis $(33,34)$. The extrinsic apoptotic pathway is activated by ligand binding at the cell surface, while the intrinsic cell death pathway is triggered by UV-irradiation, growth factor deprivation or the presence of stress stimuli, for example oxidative stress, DNA damage or endoplasmic reticulum stress, and results in the induction of the proapoptotic Bcl-2 family members. Western blotting experiments in the present study indicated that the oleuropein-induced apoptosis of HepG2 cells is caspase-dependent. Furthermore, oleuropein markedly increased BAX gene expression, while decreasing that of the $\mathrm{Bcl}-2$ gene. The alteration in the ratio of $\mathrm{BAX} / \mathrm{Bcl}-2$ is correlated with apoptosis through a mitochondrial pathway (35). These results are, in part, consistent with the mechanism of apoptosis identified in MCF-7 cells treated with oleuropein (12). The two studies characterized an apoptotic induction function of oleuropein, and the involvement of BAX and Bcl-2 in the oleuropein-induced cell apoptotic pathway. Notably, in MCF-7 breast cancer cells, oleuropein regulated the expression of p53. Further study is required to elucidate the role of p53 in liver cancer following oleuropein treatment, which may provide a clinical basis for treatment via a combination of drug delivery and gene therapy.

Oleuropein induces apoptosis in cancer cell lines via diverse pathways. In the present study, key mediators that may be involved in the apoptotic pathway were screened. It was found that the PI3K/AKT signaling pathway was markedly suppressed in HepG2 cells treated with oleuropein. Furthermore, oleuropein altered the activity of the NF- $\kappa \mathrm{B}$ and Notch pathways to a certain extent, which indicated the involvement of these pathways in this process. The PI3K/AKT pathway is known to be an important survival pathway that is activated in cancer $(36,37)$. Deregulation of the PI3K/AKT/mTOR signaling pathways has been observed in HCC (38). Activation of the PI3K/AKT pathway is associated with the promotion of invasion and migration of $\mathrm{HCC}(39,40)$. Based on the results of the current study, oleuropein may repress the phosphorylation of AKT. Indeed, the rescue experiments using transfection with a plasmid containing AKT, confirmed the involvement of AKT in apoptosis in these cells. In addition, LY294002, a specific PI3K inhibitor, increased cell death when used together with oleuropein. It may therefore be amenable for use in HCC prevention. ROS are considered to be downstream of the activation of AKT $(41,42)$. The present study showed that oleuropein treatment augmented intracellular ROS levels, and that removal of ROS increased the level of phosphorylated AKT and decreased the activation of apoptosis. This suggests that PI3K/AKT is a critical mediator in the oleuropein-induced apoptosis of HepG2 cells and that ROS are upstream of the AKT pathway. However, additional mechanisms are likely to be involved and require further investigation.

In conclusion, the current study demonstrated that oleuropein induced apoptosis in human hepatocellular carcinoma cells via suppression of PI3K/AKT, and that ROS accumulation is involved in the apoptotic pathway. Therefore, oleuropein may have potential as a novel agent in the prevention and treatment of liver cancer.

\section{References}

1. Llovet JM, Burroughs A and Bruix J: Hepatocellular carcinoma. Lancet 362: 1907-1917, 2003.

2. Singh S, Singh PP, Roberts LR and Sanchez W: Chemopreventive strategies in hepatocellular carcinoma. Nat Rev Gastroenterol Hepatol 11: 45-54, 2013

3. Cheng JW and Lv Y: New progress of non-surgical treatments for hepatocellular carcinoma. Med Oncol 30: 381, 2013.

4. Bruix J and Sherman M; American Association for the Study of Liver Diseases: Management of hepatocellular carcinoma: an update. Hepatology 53: 1020-1022, 2011.

5. Bravi F, Bosetti C, Tavani A, Gallus S and La Vecchia C: Coffee reduces risk for hepatocellular carcinoma: an updated meta-analysis. Clin Gastroenterol Hepatol 11: 1143-1421, 2013.

6. Calvisi DF, Ladu S, Hironaka K, Factor VM and Thorgeirsson SS: Vitamin E down-modulates iNOS and NADPH oxidase in c-Myc/TGF-alpha transgenic mouse model of liver cancer. J Hepatol 41: 815-822, 2004.

7. Sawada N, Inoue M, Iwasaki M, et al: Consumption of n-3 fatty acids and fish reduces risk of hepatocellular carcinoma. Gastroenterology 142: 1468-1475, 2012.

8. Hursting SD, Thornquist M and Henderson MM: Types of dietary fat and the incidence of cancer at five sites. Prev Med 19: 242-253, 1990.

9. Liu K, Stamler J, Moss D, Garside D, Persky V and Soltero I: Dietary cholesterol, fat, and fibre, and colon-cancer mortality. An analysis of international data. Lancet 2: 782-785, 1979.

10. Ferro-Luzzi A and Sette S: The Mediterranean Diet: an attempt to define its present and past composition. Eur J Clin Nutr 43 Suppl 2: 13-29, 1989.

11. Andreadou I, Mikros E, Ioannidis K, et al: Oleuropein prevents doxorubicin-induced cardiomyopathy interfering with signaling molecules and cardiomyocyte metabolism. J Mol Cell Cardiol 69: 4-16, 2014

12. Hassan ZK, Elamin MH, Omer SA, et al: Oleuropein induces apoptosis via the p53 pathway in breast cancer cells. Asian Pac J Cancer Prev 14: 6739-6742, 2013.

13. Bulotta S, Corradino R, Celano M, et al: Antioxidant and antigrowth action of peracetylated oleuropein in thyroid cancer cells. J Mol Endocrinol 51: 181-189, 2013.

14. Cardeno A, Sánchez-Hidalgo M, Rosillo MA and Alarcón de la Lastra C: Oleuropein, a secoiridoid derived from olive tree, inhibits the proliferation of human colorectal cancer cell through downregulation of HIF-1 $\alpha$. Nutr Cancer 65: 147-156, 2013.

15. Cotter TG: Apoptosis and cancer: the genesis of a research field. Nat Rev Cancer 9: 501-507, 2009.

16. Johnstone RW, Ruefli AA and Lowe SW: Apoptosis: a link between cancer genetics and chemotherapy. Cell 108: 153-164, 2002.

17. Kumar A, Rajendran V, Sethumadhavan R and Purohit R: AKT kinase pathway: a leading target in cancer research. Scientific World Journal 2013: 756134, 2013.

18. Pavlidou A and Vlahos NF: Molecular alterations of $\mathrm{PI} 3 \mathrm{~K} / \mathrm{Akt} / \mathrm{mTOR}$ pathway: a therapeutic target in endometrial cancer. Scientific World Journal 2014: 709736, 2014.

19. Franke TF, Hornik CP, Segev L, Shostak GA and Sugimoto C: PI3K/Akt and apoptosis: size matters. Oncogene 22: 8983-8998, 2003.

20. Datta SR, Dudek H, Tao X, et al: Akt phosphorylation of BAD couples survival signals to the cell-intrinsic death machinery. Cell 91: 231-241, 1997.

21. Hahn-Windgassen A, Nogueira V, Chen CC, Skeen JE, Sonenberg N and Hay N: Akt activates the mammalian target of rapamycin by regulating cellular ATP level and AMPK activity. J Biol Chem 280: 32081-32089, 2005. 
22. Rokutanda S, Fujita T, Kanatani N, et al: Akt regulates skeletal development through GSK3, mTOR, and FoxOs. Dev Biol 328: 78-93, 2009.

23. Adams JM and Cory S: Life-or-death decisions by the Bcl-2 protein family. Trends Biochem Sci 26: 61-66, 2001.

24. Nicholson KM and Anderson NG: The protein kinase B/Akt signalling pathway in human malignancy. Cell Signal 14: 381-395, 2002.

25. Vlahos CJ, Matter WF, Hui KY and Brown RF: A specific inhibitor of phosphatidylinositol 3-kinase, 2-(4-morpholinyl)-8-phenyl-4H-1-benzopyran-4-one(LY294002). J Biol Chem 269: 5241-5248, 1994.

26. Nogueira V, Park Y, Chen CC, et al: Akt determines replicative senescence and oxidative or oncogenic premature senescence and sensitizes cells to oxidative apoptosis. Cancer Cell 14: 458-470, 2008.

27. Robey RB and Hay N: Is Akt the 'Warburg kinase'? - Akt-energy metabolism interactions and oncogenesis. Semin Cancer Biol 19: 25-31, 2009.

28. Fini L, Hotchkiss E, Fogliano V, et al: Chemopreventive properties of pinoresinol-rich olive oil involve a selective activation of the ATM-p53 cascade in colon cancer cell lines. Carcinogenesis 29: 139-146, 2008.

29. La Vecchia C: Association between Mediterranean dietary patterns and cancer risk. Nutr Rev 67 Suppl 1: S126-S129, 2009

30. Menendez JA, Papadimitropoulou A, Vellon L and Lupu R: A genomic explanation connecting 'Mediterranean diet', olive oil and cancer: oleic acid, the main monounsaturated fatty acid of olive oil, induces formation of inhibitory 'PEA3 transcription factor-PEA3 DNA binding site' complexes at the Her-2/neu (erbB-2) oncogene promoter in breast, ovarian and stomach cancer cells. Eur J Cancer 42: 2425-2432, 2006.

31. Kaufmann SH and Earnshaw WC: Induction of apoptosis by cancer chemotherapy. Exp Cell Res 256: 42-49, 2000.
32. Wiita AP, Ziv E, Wiita PJ, et al: Global cellular response to chemotherapy-induced apoptosis. Elife 29: e01236, 2013.

33. Czerski L and Nunez G: Apoptosome formation and caspase activation: is it different in the heart? J Mol Cell Cardiol 37: 643-652, 2004

34. Fulda S and Debatin KM: Apoptosis signaling in tumor therapy. Ann NY Acad Sci 1028: 150-156, 2004.

35. Zhang L, Yu J, Park BH, Kinzler KW and Vogelstein B: Role of BAX in the apoptotic response to anticancer agents. Science 290: 989-992, 2000.

36. Fresno Vara JA, Casado E, de Castro J, Cejas P, Belda-Iniesta C and González-Barón M: PI3K/Akt signalling pathway and cancer. Cancer Treat Rev 30: 193-204, 2004.

37. Hennessy BT, Smith DL, Ram PT, Lu Y and Mills GB: Exploiting the PI3K/AKT pathway for cancer drug discovery. Nat Rev Drug Discov 4: 988-1004, 2005.

38. Minguez B, Tovar V, Chiang D, Villanueva A and Llovet JM: Pathogenesis of hepatocellular carcinoma and molecular therapies. Curr Opin Gastroenterol 25: 186-194, 2009.

39. Saxena NK, Sharma D, Ding X, et al: Concomitant activation of the JAK/STAT, PI3K/AKT, and ERK signaling is involved in leptin-mediated promotion of invasion and migration of hepatocellular carcinoma cells. Cancer Res 67: 2497-2507, 2007.

40. Wang GL, Iakova P, Wilde M, Awad S and Timchenko NA: Liver tumors escape negative control of proliferation via PI3K/Akt-mediated block of C/EBP alpha growth inhibitory activity. Genes Dev 18: 912-925, 2004.

41. Nogueira V, Park Y, Chen CC, et al: Akt determines replicative senescence and oxidative or oncogenic premature senescence and sensitizes cells to oxidative apoptosis. Cancer Cell 14: 458-470, 2008.

42. Liu J, Zhou J and Xing D: Phosphatidylinositol 3-kinase plays a vital role in regulation of rice seed vigor via altering NADPH oxidase activity. PLoS One 7: e33817, 2012. 